\title{
IMOBILIZAÇÃO DA LACASE EM MICROPARTÍCULAS DE QUITOSANA OBTIDAS POR SPRAY DRYING E USADAS NA CONSTRUÇÃO DE BIOSSENSORES
}

\author{
Inês Rosane Welter Zwirtes de Oliveira* e Orlando Fatibello-Filho \\ Departamento de Química, Universidade Federal de São Carlos, CP 676, 13560-970 São Carlos -SP, Brasil \\ Suellen Cadorin Fernandes e Iolanda Cruz Vieira \\ Departamento de Química, Universidade Federal de Santa Catarina, 88040-900 Florianópolis -SC, Brasil
}

Recebido em 28/5/08; aceito em 17/12/08; publicado na web em 11/5/09

\begin{abstract}
IMMOBILIZATION OF LACCASE IN MICROPARTICLES OF CHITOSAN OBTAINED BY SPRAY DRYING AND USED IN THE BIOSENSORS CONSTRUCTION. Biosensors based on laccase immobilized on microparticles of chitosan crosslinked with tripolyphosphate (biosensor I) and glyoxal (biosensor II) obtained by spray drying for the determinations of rutin in pharmaceutical formulations were developed. Under optimized operational conditions ( $\mathrm{pH} \mathrm{4.0,} \mathrm{frequency} \mathrm{of} 30 \mathrm{~Hz}$, pulse amplitude of $40 \mathrm{mV}$ and scan increment of $2.0 \mathrm{mV}$ ) two analytical curves were obtained for both biosensors showing a detection limit of $6.2 \times 10^{-8} \mathrm{~mol} \mathrm{~L}^{-1}$ for biosensor (I) and $2.0 \times 10^{-8} \mathrm{~mol} \mathrm{~L}^{-1}$ for biosensor (II). The recovery of rutin from pharmaceutical sample ranged from 90.7 to $105.0 \%$ and the lifetime of these biosensors were 4 months (at least 400 determinations).
\end{abstract}

Keywords: spray dryer; microparticles; biosensor.

\section{INTRODUÇÃO}

A técnica de spray drying tem sido utilizada em diversos segmentos industriais devido à rápida e eficiente secagem de alimentos, produtos farmacêuticos, entre outras substâncias. ${ }^{1}$ Além do processo de desidratação é amplamente usada para microencapsulação de produtos farmacêuticos aplicados em sistemas de liberação controlada de drogas. ${ }^{1-3} \mathrm{Na}$ indústria alimentícia, a microencapsulação é empregada principalmente para melhorar a estabilidade dos alimentos, aumentando o tempo de armazenamento e mantendo o valor nutritivo. ${ }^{1-4} \mathrm{~A}$ técnica oferece diversas vantagens, tais como baixo custo de operação, alta qualidade das micropartículas produzidas, capacidade de processar diversas matérias-prima, produção de micropartículas com elevada área superficial, processo de etapa única e apresenta flexibilidade na formulação do material empregado. ${ }^{1-5}$ Entre as principais desvantagens pode ser citada a limitação na escolha do encapsulante que, preferencialmente, deve apresentar baixa viscosidade em altas concentrações. Os fatores de formulação e os parâmetros selecionados no equipamento determinam as propriedades das micropartículas produzidas. ${ }^{1-5}$ Entre os polímeros mais usados na produção de micropartículas por spray drying podem ser citados quitosana, quitina, dextrana, albumina, colágeno, celulose e derivados, amido e derivados, pectina, alginato, cloreto de polivinila e polimetilacrilato. ${ }^{4-6} \mathrm{O}$ processo por spray drying consiste na nebulização de materiais no estado fluido (solução, emulsão ou pasta) dentro de uma câmara submetida a uma corrente controlada de ar quente. $O$ contato das gotículas produzidas com o ar quente promove a evaporação do solvente, obtendo-se o material em pó. ${ }^{1}$

Enzimas imobilizadas têm sido amplamente empregadas em indústrias químicas, farmacêuticas e alimentícias, por favorecerem principalmente a reutilização e aumentarem a estabilidade, diminuindo assim os custos do processo. Suportes como microesferas poliméricas são alternativas favoráveis para imobilização de enzimas, pois apresentam facilidade na obtenção, são produzidas com uma grande variedade de composições e podem ser modificadas por diversos métodos de ativação. Além disso, as microesferas apresentam

*e-mail: inesrz1@yahoo.com.br grande área superficial e, dependendo do processo de reticulação do polímero, podem apresentar maior porosidade, aumentando a capacidade para imobilização de enzimas. ${ }^{7,8}$ A quitosana, ( $\beta(1-4)-2$ amino-2-deoxi-D-glicose), possui um grupo amino primário e dois grupos hidroxila livres em cada unidade glicosamina, que podem ser modificados por agentes reticulantes.$^{6-14}$ Ligações do tipo base de Schiff são formadas entre os grupos amino da quitosana e os grupos aldeídos de agentes reticulantes, tais como glutaraldeído, glioxal, D,L gliceraldeído, formaldeído, entre outros. ${ }^{6-14}$ Tem sido demonstrado que as imobilizações de enzimas por ligação covalente aumentam a resistência à desnaturação em condições extremas de $\mathrm{pH}$, temperatura ou, ainda, em alguns casos, em presença de solventes orgânicos. ${ }^{7,8}$ Outro processo de ativação da quitosana é realizado em meio ácido, sendo os grupos amino da quitosana protonados e essas cargas positivas formadas podem interagir com diversos ânions como, por exemplo, tripolifosfato de sódio, sulfato de sódio, citrato de sódio, alginato de sódio, entre outros. ${ }^{6,9}$

As lacases (EC 1.10.3.2) pertencem à classe das oxiredutases e são encontradas em plantas, insetos, bactérias e fungos. Embora os centros de cobre sejam similares para todas as lacases de fungos, diferenças significativas são observadas nas propriedades termodinâmicas e cinéticas em função do microorganismo de origem. ${ }^{15-17}$ As lacases são usadas em diversas aplicações industriais, incluindo biorremediação, clarificação de vinho, análise de drogas, produção de etanol e construção de biossensores. ${ }^{18,19}$ Vários biossensores contendo lacase imobilizada têm sido desenvolvidos para a determinação de compostos fenólicos. ${ }^{20-24}$

A rutina, Figura 1, é um derivado de flavona e como resultado da atividade farmacológica é empregada em fármacos de efeito antiinflamatório, antitumor, antialérgico, antiviral, entre outras propriedades..$^{25-27}$ Diversos procedimentos são empregados para a determinação deste flavonóide, entre eles os métodos espectrofotométricos, ${ }^{28-30}$ eletroforese capilar $^{31,32}$ e os eletroquímicos. ${ }^{33-35}$ Os métodos espectrofotométricos têm sido usados para a determinação individual de rutina e também simultânea de outros flavonóides. ${ }^{28-30}$ Os métodos eletroquímicos utilizando biossensores apresentaram boas figuras de mérito para a determinação de flavonóides em produtos farmacêuticos. ${ }^{33-35}$

O presente trabalho teve como objetivo imobilizar a lacase em micropartículas de quitosana reticuladas com glioxal e com tripoli- 


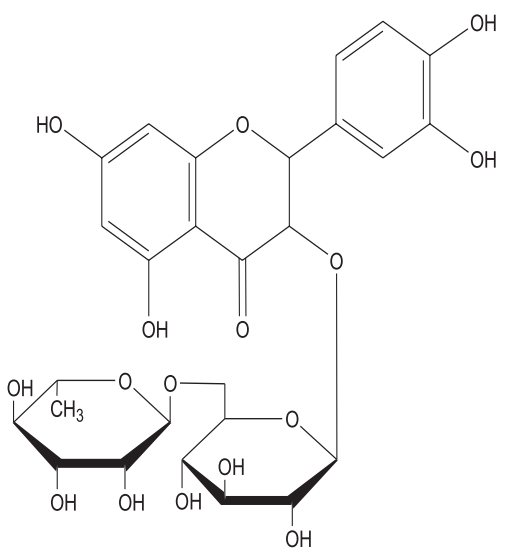

Figura 1. Estrutura química da rutina

fosfato de sódio (TPP) obtidas por spray drying, visando a construção de biossensores para determinação de rutina em produtos farmacêuticos. Após imobilização, os suportes contendo lacase foram usados na construção de biossensores e parâmetros analíticos, tais como concentração da enzima, $\mathrm{pH}$ e os de voltametria de onda quadrada (frequiência, amplitude de potencial e incremento), foram investigados. A resposta e a eficiência dos biossensores desenvolvidos foram verificadas na determinação de rutina em produtos farmacêuticos.

\section{PARTE EXPERIMENTAL}

\section{Soluções e reagentes}

Reagentes de grau analítico foram usados para preparar as soluções. Lacase (Aspergillus oryzae) obtida da Novozymes ${ }^{\circledR}$ (Denilite ${ }^{\circledR}$ II BASE), pó de grafite da Fisher Scientific (partícula $<50 \mu \mathrm{m} \mathrm{e} \geq$ 99,5\%) e Nujol da Aldrich foram empregados para construção dos biossensores.

Tripolifosfato de sódio (TPP) e glioxal foram obtidos da Sigma e usados para a reticulação da quitosana. A quitosana foi obtida da Sigma, com grau de desacetilação de 85\%. Ácido 2,2"-Azino-bis (3-etilbenzetiazolino-6-sulfônico) (ABTS) usado para determinação da atividade da lacase, cloreto de sódio, amido, polietilenoglicol, estearato de magnésio, lactose e sacarose foram obtidos da Sigma.

Soluções tampão acetato $0,1 \mathrm{~mol} \mathrm{~L}^{-1}$ (pH 3,0 a 5,0) e soluções tampão fosfato $0,1 \mathrm{~mol} \mathrm{~L}^{-1}$ (pH 6,0 a 8,0) foram preparadas. Solução estoque de rutina $2,0 \times 10^{-4} \mathrm{~mol} \mathrm{~L}^{-1}$ foi preparada em etanol e tampão acetato $0,1 \mathrm{~mol} \mathrm{~L}^{-1}, \mathrm{pH} 4,0$ na proporção $30: 70 \%$ (v/v), respectivamente. Soluções padrão de $2,0 \times 10^{-7}$ a $1,3 \times 10^{-5} \mathrm{~mol} \mathrm{~L}^{-1}$ foram preparadas pela diluição apropriada da solução estoque com tampão acetato 0,1 mol L-1, $\mathrm{pH} 4,0$.

\section{Equipamentos e eletrodos}

As medidas espectrofotométricas para a determinação de atividade da lacase foram obtidas usando-se um espectrofotômetro B572 (Micronal) e cubeta de quartzo de $1,00 \mathrm{~cm}$ de caminho óptico. As microesferas de quitosana reticuladas com TPP e glioxal foram obtidas usando-se um mini spray dryer (Büchi ${ }^{\circledR}$ B-191, Suíça). A morfologia das microesferas de quitosana reticulada foi examinada por microscopia eletrônica de varredura em um microscópio da Philips (Modelo XL 30).

As medidas de voltametria de onda quadrada foram obtidas utilizando-se um potenciostato/galvanostato da Autolab modelo PGSTAT12 e uma célula de vidro com capacidade aproximada de $15 \mathrm{~mL}$. Os biossensores (I) e (II) contendo lacase imobilizada em micropartículas de quitosana reticuladas com TPP e glioxal, respectivamente, foram usados como eletrodos de trabalho. Uma placa de platina com área de $0,5 \mathrm{~cm}^{2}$ foi usada como contra-eletrodo e como eletrodo de referência foi utilizado um eletrodo de prata-cloreto de prata, $\mathrm{Ag} / \mathrm{AgCl}\left(\mathrm{KCl} \mathrm{3,0} \mathrm{mol} \mathrm{L}^{-1}\right)$.

\section{Determinação da atividade da lacase}

A atividade da lacase livre foi determinada usando-se o ABTS como substrato, sendo o produto da reação enzimática medida em $420 \mathrm{~nm}$. Neste método empregaram-se 2,8 $\mathrm{mL}$ de ABTS $0,5 \mathrm{mmol}$ e $0,2 \mathrm{~mL}$ de lacase em tampão acetato $0,1 \mathrm{~mol} \mathrm{~L}^{-1}, \mathrm{pH} 5,0$. Uma unidade de atividade da lacase é definida como a quantidade de enzima necessária para oxidar $1 \mu \mathrm{mol}$ de ABTS por minuto. ${ }^{36,37}$

\section{Obtenção das micropartículas de quitosana reticuladas com TPP ou com glioxal}

Para a obtenção das micropartículas de quitosana reticuladas com TPP, foram adicionados $10 \mathrm{~mL}$ de TPP $2 \%(\mathrm{~m} / \mathrm{v})$ em $100 \mathrm{~mL}$ de quitosana $1 \%(\mathrm{~m} / \mathrm{v})$ dissolvido em ácido acético $5 \%(\mathrm{v} / \mathrm{v})$. Da mesma forma, adicionou-se $1,0 \mathrm{~mL}$ de glioxal $40 \%$ (v/v) em $100 \mathrm{~mL}$ de solução de quitosana $1 \%(\mathrm{~m} / \mathrm{v})$. A seguir, cada solução foi homogeneizada durante 30 min com agitação mecânica e nebulizada por spray dryer com temperatura de entrada de $180^{\circ} \mathrm{C}$, fluxo do ar de $7 \%$ ( $2 \mathrm{~mL} / \mathrm{min}$ ), taxa de aspiração de $500 \mathrm{NL} / \mathrm{h}$ e pressão do ar de $5 \mathrm{~kg} /$ $\mathrm{cm}^{3}$. As emulsões de quitosana contendo TPP e glioxal foram obtidas por spray drying separadamente e as micropartículas obtidas foram usadas na construção dos biossensores (I) e (II), respectivamente.

\section{Imobilização da lacase e construção dos biossensores}

As micropartículas de quitosana reticuladas foram usadas como suporte sólido para imobilização da lacase. Foram adicionadas alíquotas de 10 a $50 \mu \mathrm{L}$ de lacase $\left(0,14\right.$ a 0,68 unidades $\left.\mathrm{mL}^{-1}\right)$ sobre $0,1 \mathrm{~g}$ de micropartículas de quitosana. Após $2 \mathrm{~h}$ de imobilização com agitação manual, os suportes foram lavados com solução tampão acetato $0,1 \mathrm{~mol}$ $\mathrm{L}^{-1}$ (pH 5,0), para remover o excesso de enzima. Após secos, os suportes contendo lacase imobilizada foram armazenados em dessecador.

Os biossensores foram construídos a partir da maceração, por $20 \mathrm{~min}$, de $375 \mathrm{mg}$ de pó de grafite e $50 \mathrm{mg}$ de micropartículas de quitosana previamente reticuladas contendo 0,55 unidades $\mathrm{mL}^{-1} \mathrm{de}$ lacase imobilizada. Em seguida, adicionaram-se $75 \mathrm{mg}$ de Nujol e macerou-se por mais $20 \mathrm{~min}$. A pasta obtida foi transferida para uma seringa de $1 \mathrm{~mL}$ e um fio de cobre foi usado como contato elétrico. ${ }^{10-13}$ Os biossensores foram armazenados a temperatura ambiente e aplicados como eletrodos de trabalho.

\section{Medidas eletroquímicas e aplicação dos biossensores}

As medidas de voltametria de onda quadrada foram realizadas varrendo-se o potencial de $+0,60 \mathrm{a}+0,10 \mathrm{~V} v s \mathrm{Ag} / \mathrm{AgCl}(\mathrm{KCl} 3,0 \mathrm{~mol}$ $\left.\mathrm{L}^{-1}\right)$. Os estudos de freqüência $(10 \mathrm{a} 100 \mathrm{~Hz})$, amplitude de potencial $(10 \mathrm{a} 100 \mathrm{mV})$ e incremento $(10 \mathrm{a} 100 \mathrm{mV})$ foram investigados.

Os biossensores construídos foram utilizados na determinação de rutina em produtos farmacêuticos (amostras A, B e C). Inicialmente, foram triturados 10 comprimidos, pesados em triplicata $(50 \mathrm{mg})$ e dissolvidos em solução tampão acetato $0,1 \mathrm{~mol} \mathrm{~L}^{-1}, \mathrm{pH} 4,0$ e etanol na proporção 70:30\% (v/v), respectivamente. Quando necessário, as amostras foram sonificadas em banho de ultra-som durante 5 min e filtradas. Para a amostra líquida (C), 1,0 mL do produto farmacêutico foi diluído em $50 \mathrm{~mL}$ de solução contendo etanol:tampão acetato 0,1 mol L ${ }^{-1}, \mathrm{pH} 4,0$ (30:70\% v/v). 
O método de adição de padrão foi usado para determinar o teor de recuperação e a concentração de rutina nos produtos farmacêuticos. Neste estudo, 1,0 mL das soluções de cada amostra comercial foi transferido para uma célula eletroquímica contendo $10,0 \mathrm{~mL}$ de solução tampão acetato $0,1 \mathrm{~mol} \mathrm{~L}^{-1}, \mathrm{pH} 4,0$. Em seguida, foram realizadas sucessivas adições da solução de rutina padrão. Após cada adição, foram registrados os voltamogramas de onda quadrada no intervalo de potencial de $+0,60 \mathrm{a}+0,10 \mathrm{~V}$, frequiência de $30 \mathrm{~Hz}$, amplitude de potencial de $40 \mathrm{mV}$ e incremento de 2,0 mV. Todas as medidas usando os biossensores propostos foram realizadas em triplicata. Com objetivo de comparar os valores obtidos de rutina nos produtos farmacêuticos usando os biossensores propostos, foi usado o método espectrofotométrico da $\mathrm{AOAC}^{38}$ que emprega solução de ácido acético:etanol na proporção 10:90\% (v/v) e detecção em comprimento de onda de $366 \mathrm{~nm}$.

\section{RESULTADOS E DISCUSSÃO}

\section{Morfologia das micropartículas de quitosana reticuladas com TPP ou com glioxal}

As micropartículas de quitosana reticuladas com TPP e glioxal foram obtidas por spray drying e a morfologia examinada por microscopia eletrônica de varredura (MEV), como mostradas nas Figuras $2 \mathrm{a}$ e $2 \mathrm{~b}$, respectivamente.

As micropartículas reticuladas com TPP, Figura 2a, apresentaram morfologia não esférica com cavidades e tamanho variando de 3,0 a $8,9 \mu \mathrm{m}$ e foram empregadas como suporte para imobilização da lacase e construção do biossensor (I). Shu e Zhu ${ }^{39}$ obtiveram micropartículas de quitosana reticuladas com TPP com morfologia similar às obtidas neste trabalho.

As micropartículas de quitosana reticuladas com glioxal, Figura 2b, apresentaram superfície lisa, forma esférica com tamanho variando de 0,9 a $6,7 \mu \mathrm{m}$ e foram usadas como suporte para imobilização da lacase e construção do biossensor (II). Micropartículas com tamanho e morfologia similares foram reportadas por Desay e Park. ${ }^{40} \mathrm{O}$ processo de reticulação da quitosana é de grande importância, pois, além de promover a formação de uma rede polimérica na qual a enzima pode estar covalentemente ligada e/ou entrapeada, previne o intumescimento das micropartículas de quitosana em soluções aquosas, ${ }^{41}$ favorecendo uma maior estabilidade da enzima imobilizada.

A morfologia não esférica observada para micropartículas de quitosana reticuladas com TPP está relacionada principalmente com a concentração e natureza do agente reticulante. ${ }^{39,40}$ Esse comportamento foi comprovado por alguns pesquisadores, ${ }^{39,40}$ que realizaram estudos sistemáticos variando a concentração deste agente de reticulação e verificaram que micropartículas de quitosana reticuladas com TPP em excesso, obtidas por spray drying, apresentaram morfologia similar às apresentadas neste trabalho.

\section{Imobilização da lacase em microesferas de quitosana reticuladas com TPP ou com glioxal}

As propriedades e a eficiência das enzimas imobilizadas são dependentes principalmente do tipo de suporte empregado no processo da imobilização. A quitosana é um polissacarídeo de origem natural que apresenta diversas características, tais como facilidade para formação de gel, hidrofóbica, biodegradável, biocompatível, antibactericida, mucoadesiva, entre outras. Além disso, possui grupos funcionais que são susceptíveis a modificações químicas, facilitando a imobilização de enzimas. A reticulação da quitosana e a imobilização de enzimas neste suporte são amplamente reportadas na literatura. ${ }^{10-14,42-44}$

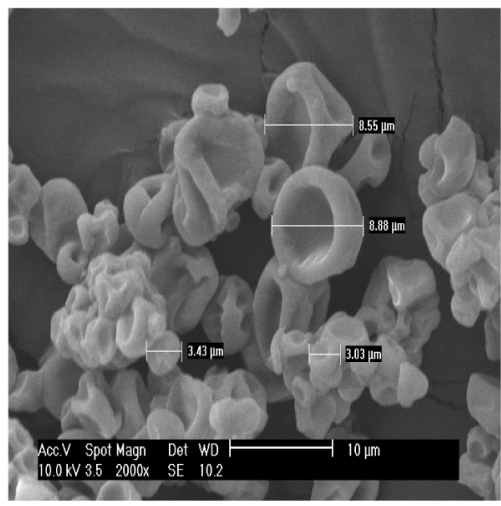

(a)

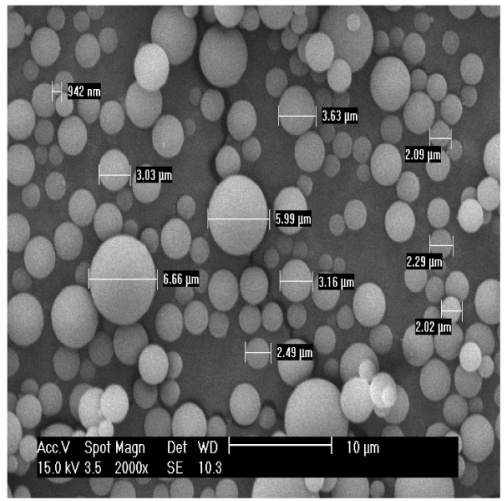

(b)

Figura 2. Microscopia eletrônica de varredura (MEV) das micropartículas de quitosana obtidas por spray drying, reticuladas com: (a) tripolifosfato de sódio $(T P P)$ e (b) glioxal

A Figura 3 mostra uma proposta da imobilização da lacase em quitosana (a) reticulada com TPP (b) baseada na literatura. ${ }^{10-14,42-44}$ O TPP é um íon pentavalente e reage com a quitosana formando ligações iônicas entre as cargas negativas dos oxigênios do TPP $\left(\mathrm{P}_{3} \mathrm{O}_{10}{ }^{5-}\right)$ com os grupos amino protonados da quitosana $\left(\mathrm{NH}_{3}^{+}\right) \mathrm{em}$ valores de $\mathrm{pH}$ menores que 6,5, obtendo-se a estrutura representada em (c). Sugere-se que neste processo a lacase (d) esteja entrapeada entre as ligações iônicas da quitosana e do TPP, resultando na estrutura apresentada em (e).

Os grupos amino da quitosana (a), Figura 4, reagem com os grupos carbonilas ativos do glioxal (b), gerando $\alpha$-hidroxi-aminas mostrados nas estruturas representadas em (c, d). As $\alpha$-hidroxiaminas derivadas de aminas primárias sofrem, usualmente, desidratação espontânea em meio ácido, para gerar iminas substituídas ou ligações do tipo base de Schiff ${ }^{10-14,42-44}(\mathrm{e})$, gerando uma rede polimérica entre a quitosana e o glioxal (f). A lacase (g) pode estar ligada ao suporte de quitosana reticulada com glioxal por ligações covalentes dos grupos amino da enzima, com os grupos aldeídicos do glioxal que não reagiram e ainda se encontram entrapeados nos espaços intersticiais formados entre o biopolímero e o glioxal, como mostra o esquema (h).

\section{Otimização dos biossensores}

Os biossensores contendo a enzima lacase imobilizada em micropartículas de quitosana reticuladas foram otimizados, estudando-se a composição da pasta de carbono (grafite/Nujol), o efeito da concentração da enzima, pH e os parâmetros da voltametria de onda quadrada (frequiência, amplitude de potencial e incremento).

A composição de grafite/Nujol na construção dos biossensores 


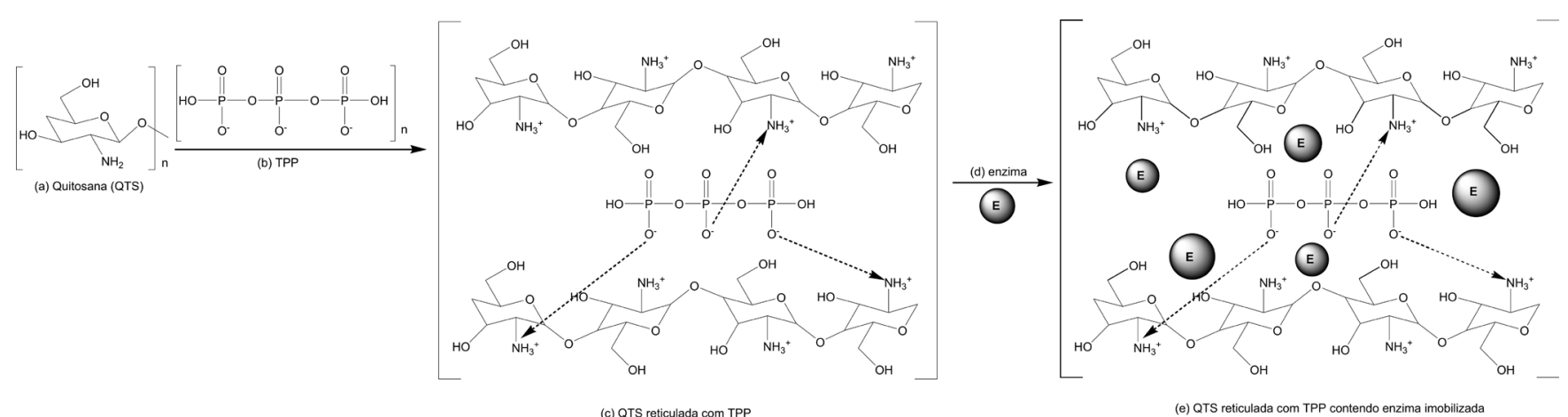

Figura 3. Esquema proposto para imobilização da lacase em quitosana reticulada com tripolifosfato
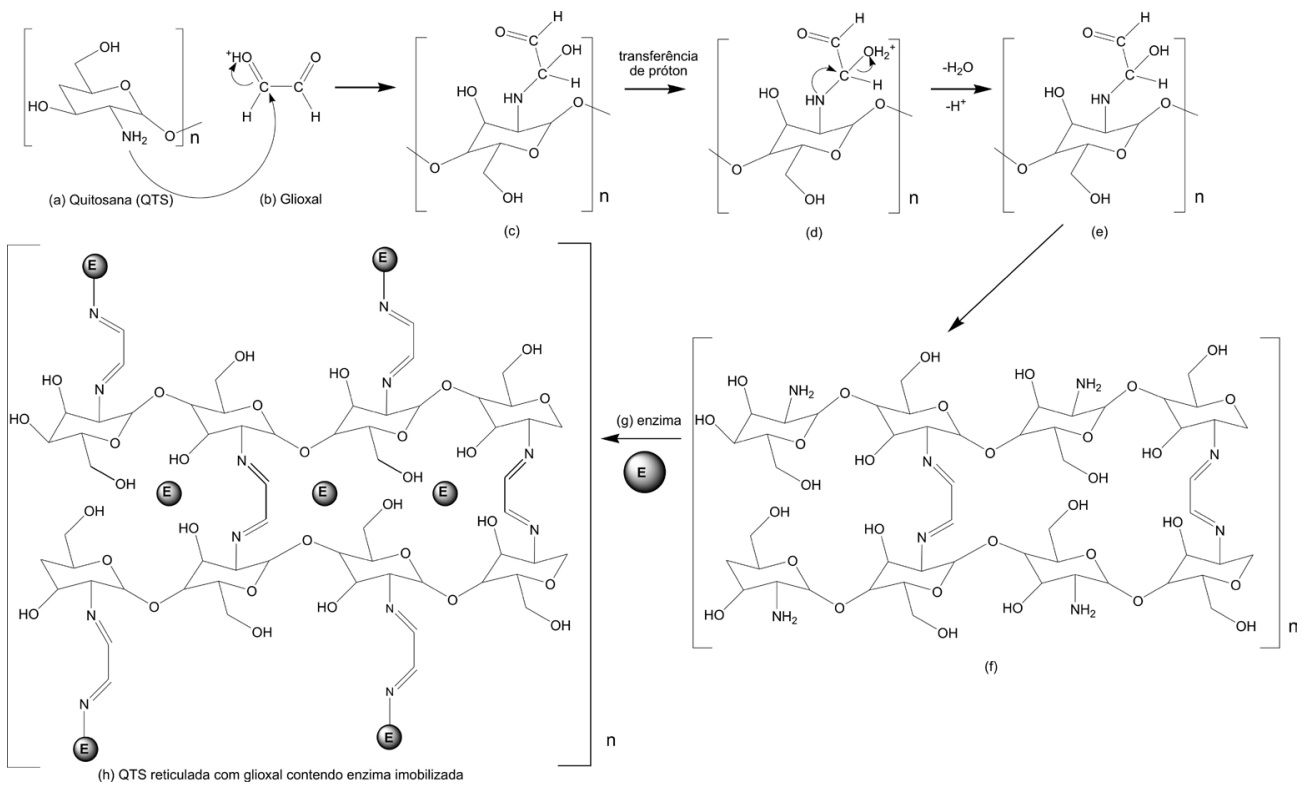

Figura 4. Esquema proposto para imobilização da lacase em quitosana reticulada com glioxal

foi avaliada a partir da proporção de 65/25; 70/20;75/15; 80/10\% $(\mathrm{m} / \mathrm{m})$ de grafite/Nujol e mantendo-se constante o percentual de $10 \%$ de micropartículas de quitosana (contendo 5,5 unidades $\mathrm{mL}^{-1}$ de lacase). Após construção, a resposta dos biossensores foi estudada medindo-se a corrente resultante por voltametria de onda quadrada em solução de rutina $5,8 \times 10^{-6} \mathrm{~mol} \mathrm{~L} \mathrm{~L}^{-1}$. O maior sinal analítico foi obtido empregando-se os biossensores (I) e (II) com composição de 75/15/10\% (m/m) de grafite/Nujol/lacase imobilizada em quitosana reticulada. Sendo assim, essa composição de pasta de carbono foi selecionada para a construção e aplicação dos biossensores.

A atividade da lacase livre foi determinada pelo método espectrofotométrico. ${ }^{38}$ Após este estudo, foi possível avaliar a resposta dos biossensores construídos a partir de diferentes concentrações da enzima $\left(0,14 ; 0,27 ; 0,41 ; 0,55\right.$ e 0,68 unidades $\mathrm{mL}^{-1}$ de lacase) imobilizada na quitosana. As maiores respostas analíticas utilizando-se a voltametria de onda quadrada foram obtidas para os biossensores contendo 0,55 unidades $\mathrm{mL}^{-1}$ de lacase, sendo esta concentração de enzima selecionada para construção dos biossensores.

O efeito do $\mathrm{pH}$, no intervalo de 3,0 a 8,0, sobre a resposta dos biossensores foi estudado em solução de rutina $2,0 \times 10^{-6} \mathrm{~mol} \mathrm{~L}^{-1}$. A maior resposta analítica foi obtida em solução tampão acetato com pH 4,0, sendo selecionada essa solução tampão para os estudos posteriores.

Os parâmetros da voltametria de onda quadrada, importantes para a otimização dos biossensores, foram avaliados usando-se solução de rutina $2,0 \times 10^{-6} \mathrm{~mol} \mathrm{~L}^{-1}$ em tampão acetato $\left(0,1 \mathrm{~mol} \mathrm{~L}^{-1} ; \mathrm{pH} 4,0\right)$.
A freqüência $(f)$ de aplicação dos pulsos de potencial de 10 a $100 \mathrm{~Hz}$ foi investigada e o sinal analítico (corrente resultante) aumentou até $30 \mathrm{~Hz}$, permanecendo constante em valores maiores. Sendo assim, este valor de frequiência foi selecionado para os estudos posteriores. Em seguida, a amplitude (a) do pulso do potencial foi investigada no intervalo de 10 a $100 \mathrm{mV}$ e a maior resposta analítica foi obtida em $40 \mathrm{mV}$, sendo este valor utilizado nos estudos subseqüentes. O efeito do incremento $(\Delta E)$ de potencial de 1 a $5 \mathrm{mV}$ também foi investigado e o maior sinal analítico foi obtido em $2 \mathrm{mV}$, sendo este valor selecionado para o desenvolvimento deste trabalho. Na voltametria de onda quadrada a velocidade efetiva é o resultado do produto da frequiência de aplicação dos pulsos de potencial $(f)$ pelo incremento de varredura de potencial $(\Delta E \mathrm{~s})$. Um incremento de varredura maior pode aumentar o sinal analítico obtido e, em muitos casos, a sensibilidade do método, mas por outro lado, pode proporcionar alargamentos nos picos obtidos e, conseqüentemente, diminuir a resolução do método. Um bom compromisso foi alcançado com incrementos de potencial de $2 \mathrm{mV}$. A Tabela 1 resume os parâmetros analíticos investigados e os maiores valores encontrados na otimização dos biossensores (I) e (II).

\section{Voltamogramas de onda quadrada e curvas analíticas dos biossensores}

Os voltamogramas de onda quadrada foram obtidos utilizando-se os biossensores (I) e (II) e as medidas foram realizadas no intervalo de 
Tabela 1. Parâmetros experimentais investigados para otimização dos biossensores I e II

\begin{tabular}{lcc}
\hline Parâmetros experimentais & Faixa estudada & Valor selecionado \\
\hline Lacase (unidades $\left.\mathrm{mL}^{-1}\right)$ & $0,14-0,68$ & 0,55 \\
$\mathrm{pH}$ & $3,0-8,0$ & 4,0 \\
Freqüência $(\mathrm{Hz})$ & $10-100$ & 30 \\
Amplitude de potencial $(\mathrm{mV})$ & $10-100$ & 40 \\
Incremento $(\mathrm{mV})$ & $1,0-5,0$ & 2,0 \\
\hline
\end{tabular}

potencial de $+0,6 \mathrm{a}+0,1 \mathrm{mV}$, nas condições previamente otimizadas. As correntes resultantes registradas após sucessivas adições de rutina padrão usando os biossensores propostos apresentaram um pico em $+0,35 \mathrm{~V} v \mathrm{~s}$ $\mathrm{Ag} / \mathrm{AgCl}\left(\mathrm{KCl} 3,0 \mathrm{~mol} \mathrm{~L}^{-1}\right)$, como pode ser observado na Figura 5.
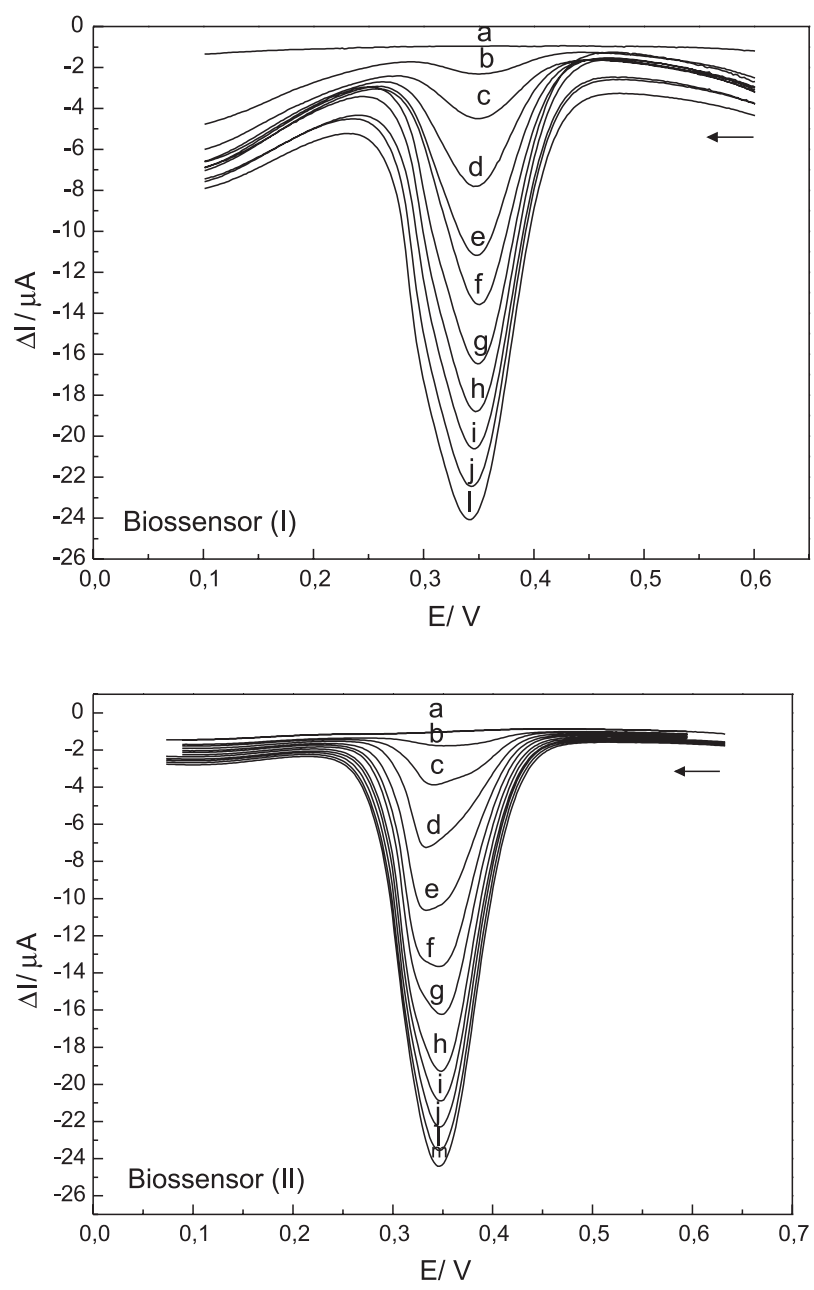

Figura 5. Voltamogramas de onda quadrada obtidos usando o biossensor (I): (a) em tampão acetato 0,1 mol L-1 $(p H 4,0)$ e adições de rutina padrão (b) $6,0 \times 10^{-7}$; (c) $9,9 \times 10^{-7}$; (d) $1,9 \times 10^{-6} ;$ (e) $2,9 \times 10^{-6} ;$ (f) $3,92 \times 10^{-6} ;(\mathrm{g}) 5,82 \times 10^{-6} ;(\mathrm{h})$

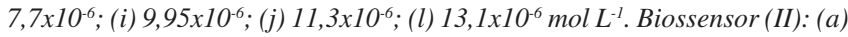

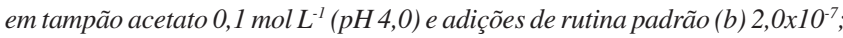

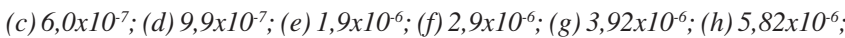

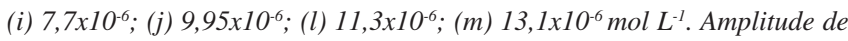
potencial de $40 \mathrm{mV}$, freqüência de $30 \mathrm{~Hz}$ e incremento de $2,0 \mathrm{mV}$

As curvas analíticas mostradas na Figura 6 foram construídas a partir das correntes resultantes $v s$ concentração de rutina. $\mathrm{O}$ biossensor (I) apresentou duas faixas lineares para rutina: (1) $6,0 \times 10^{-7} \mathrm{a}$

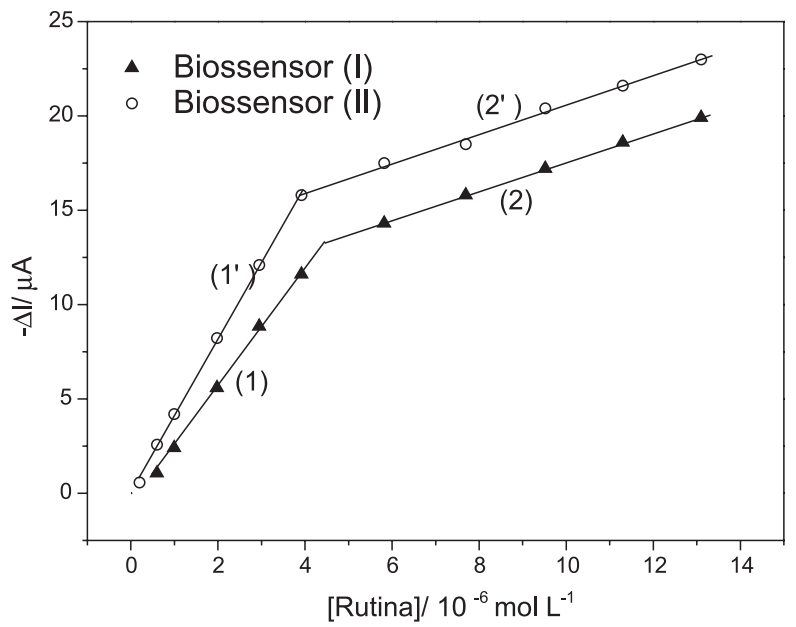

Figura 6. Curvas analíticas para rutina obtidas com os biossensores (I) e (II)

$3,9 \times 10^{-6} \mathrm{~mol} \mathrm{~L}^{-1}\left(\Delta \mathrm{I}=0,715+3,175 \times 10^{6}\right.$ [rutina], $\left.\mathrm{r}=0,9995\right) \mathrm{e}$ (2) $5,8 \times 10^{-6}$ a $1,3 \times 10^{-5} \mathrm{~mol} \mathrm{~L}^{-1}\left(\Delta \mathrm{I}=9,850+7,706 \times 10^{5}\right.$ [rutina],

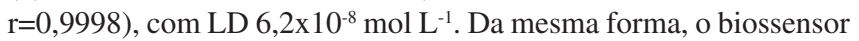
(II) apresentou duas faixas lineares correspondendo às seguintes concentrações de rutina: (1') $2,0 \times 10^{-7}$ a $3,9 \times 10^{-6} \mathrm{~mol} \mathrm{~L}^{-1}(\Delta \mathrm{I}=0,034$ $+4,064 \times 10^{6}$ [rutina]; r $\left.=0,9995\right)$ e (2') $5,8 \times 10^{-6}$ a $1,3 \times 10^{-5} \mathrm{~mol} \mathrm{~L}^{-1}$ $\left(\Delta \mathrm{I}=12,841+7,758 \times 10^{5}\right.$ [rutina]; $\left.\mathrm{r}=0,9998\right)$, com limite de detecção de $2,0 \times 10^{-8} \mathrm{~mol} \mathrm{~L}^{-1}$.

Os biossensores desenvolvidos apresentaram duas faixas lineares, e como pode ser observado nesta figura, o biossensor (II) apresentou uma maior inclinação da curva analítica no primeiro seguimento linear, e mesma inclinação no segundo segmento. A maior sensibilidade observada para este biossensor pode ser em decorrência da eficiência na formação da rede polimérica altamente organizada entre a quitosana e o glioxal, onde a enzima pode estar confinada e/ou imobilizada por ligações covalentes. ${ }^{7,8,42}$ Além disso, as micropartículas obtidas no processo de reticulação usando o glioxal apresentaram uma morfologia esférica e, possivelmente, uma maior área superficial quando comparada com as partículas obtidas pelo processo de reticulação da quitosana com TPP. Porém, como a região linear das duas curvas analíticas foi praticamente a mesma, possivelmente foi imobilizada a mesma concentração da enzima lacase nos dois suportes de quitosana. $\mathrm{O}$ fato dos biossensores apresentarem duas faixas lineares pode estar relacionado com a saturação dos sítios ativos da enzima imobilizada na superfície e no interior das micropartículas e/ou com a acessibilidade diferenciada do analito nas micropartículas. Os biossensores desenvolvidos a partir da reticulação da quitosana com TPP e com glioxal, contendo lacase imobilizada, apresentaram maior estabilidade e sensibilidade, quando comparados com os biossensores empregando quitosana reticulada com outros agentes de reticulação. ${ }^{10-13}$

\section{Repetibilidade, reprodutibilidade entre sensores, estabilidade e seletividade}

O estudo da repetibilidade foi feito individualmente, utilizandose os biossensores propostos e 9 medidas sucessivas em solução de rutina $2,0 \times 10^{-6} \mathrm{~mol} \mathrm{~L}^{-1} \mathrm{em}$ tampão acetato $0,1 \mathrm{~mol} \mathrm{~L}^{-1}(\mathrm{pH} 4,0)$ foram obtidas. O desvio padrão relativo encontrado foi menor que $1,0 \%$ para os biossensores construídos.

Para avaliar a reprodutibilidade entre os sensores, três biossensores foram construídos para cada procedimento de reticulação da quitosana com subseqüente imobilização da lacase. As medidas foram obtidas em triplicata em solução de rutina $2,0 \times 10^{-6} \mathrm{~mol} \mathrm{~L}^{-1} \mathrm{e}$ em tampão fosfato $0,1 \mathrm{~mol} \mathrm{~L}^{-1}, \mathrm{pH} 4,0$. O desvio padrão relativo (RSD) 
Tabela 2. Biossensores contendo lacase para determinação de compostos fenólicos

\begin{tabular}{|c|c|c|c|c|c|}
\hline Eletrodo & Substrato & Linearidade $\left(\mathrm{mol} \mathrm{L}^{-1}\right)$ & $\mathrm{LD}\left(\mathrm{mol} \mathrm{L}^{-1}\right)$ & Estabilidade(dias) & Ref \\
\hline \multirow[t]{3}{*}{ Platina } & Catecol & $1,0 \times 10^{-5}$ a $11 \times 10^{-5}$ & - & 38 & \multirow[t]{3}{*}{42} \\
\hline & Catequina & $1,0 \times 10^{-5}$ a $6,0 \times 10^{-5}$ & - & 38 & \\
\hline & Ácido cafêico & $1,0 \times 10^{-5}$ a $8,0 \times 10^{-5}$ & - & 38 & \\
\hline Carbono vítreo & Catecol & $0,5 \times 10^{-3}$ a $8,0 \times 10^{-4}$ & $0,3 \times 10^{-3}$ & 60 & 43 \\
\hline \multirow{2}{*}{ Carbono vítreo } & Guaiacol & $1,0 \times 10^{-6}$ a $10 \times 10^{-6}$ & $1,1 \times 10^{-7}$ & - & 44 \\
\hline & Cloroguaiacol & $1,0 \times 10^{-6}$ a $10 \times 10^{-6}$ & $2,3 \times 10^{-7}$ & & \multirow[t]{2}{*}{45} \\
\hline Carbono vítreo & Hidroquinona & $1,0 \times 10^{-7}$ a $3,0 \times 10^{-6}$ & $3,5 \times 10^{-8}$ & - & \\
\hline Pasta de carbono & Rutina & $6,0 \times 10^{-7}$ a $1,3 \times 10^{-5}$ & $6,2 \times 10^{-8}$ & 120 & $\begin{array}{c}\text { Biossensor }(\mathrm{I}) \\
\text { proposto }\end{array}$ \\
\hline Pasta de carbono & Rutina & $2,0 \times 10^{-7}$ a $1,3 \times 10^{-5}$ & $2,0 \times 10^{-8}$ & 120 & $\begin{array}{l}\text { Biossensor (I) } \\
\text { proposto }\end{array}$ \\
\hline
\end{tabular}

de 3,0 e 3,3\% foi encontrado para os biossensores (I) e (II), respectivamente. Os resultados obtidos indicam uma boa reprodutibilidade entre a construção dos biossensores empregando lacase imobilizada em micropartículas de quitosana reticuladas com TPP ou glioxal.

No estudo da estabilidade, cada biossensor foi armazenado a temperatura ambiente e testado de 15-15 dias por um período de 120 dias (400 determinações). Após este período os biossensores propostos mantiveram $93 \%$ da sua resposta inicial.

Para avaliar o efeito de substâncias concomitantes presentes nas amostras foi verificada a influência de algumas substâncias comumente encontradas em formulações farmacêuticas, tais como cloreto de sódio, amido, polietilenoglicol, estearato de magnésio, lactose e sacarose individualmente, usando-se os biossensores propostos nas proporções molares de 0,$1 ; 1,0$ e 10,0 em relação à rutina $2,2 \times 10^{-6} \mathrm{~mol} \mathrm{~L}^{-1}$ em tampão fosfato $0,1 \mathrm{~mol} \mathrm{~L}^{-1}(\mathrm{pH} 4,0)$. Nenhuma dessas substâncias investigadas causou interferência na resposta analítica dos biossensores.

Biossensores contendo lacase foram amplamente desenvolvidos e reportados na lituratura. ${ }^{45-48} \mathrm{~A}$ Tabela 2 apresenta alguns parâmetros analíticos como linearidade, limite de detecção, estabilidade dos biossensores contendo lacase imobilizada descritos na literatura e os encontrados no presente trabalho empregando os biossensores (I) e (II). Como se pode observar, as figuras de mérito obtidas com os biossensores desenvolvidos são semelhantes às reportados na literatura, ressaltando-se que um maior tempo de vida é apresentado pelos biossensores propostos.

\section{Estudos de adição de padrão e aplicação dos biossensores}

A adição múltipla de padrão é comumente utilizada na química analítica para análises de diversas amostras por se tratar de um método versátil e de fácil utilização. Além de se determinar o percentual de recuperação do analito e verificar o efeito de matriz, é possível determinar a concentração do analito na amostra. Portanto, neste trabalho, o estudo de adição de padrão nos produtos farmacêuticos foi empregado para confirmar os resultados anteriormente obtidos no estudo dos interferentes e determinar a concentração de rutina nas amostras comerciais. Neste estudo, as concentrações 14,41; 21,61 e 28,82 $\mathrm{mg} \mathrm{L}^{-1}$ de rutina padrão foram adicionadas nas amostras dos fármacos. A cada adição foram registrados os voltamogramas de onda quadrada e a partir do $\Delta I$ de cada voltamograma foi possível calcular o valor de recuperação de rutina padrão. Os resultados obtidos nestes experimentos, mostrados na Tabela 3, variaram de 90,8 a 105,0\% para o biossensor (I) e de 95,2 a $103,4 \%$ para o biossensor (II). Os resultados obtidos confirmam que o procedimento analítico proposto não sofre interferência das matrizes das amostras. A Tabela 4 apresenta uma comparação dos teores de rutina
Tabela 3. Recuperação de rutina padrão em produtos farmacêuticos usando os biossensores

\begin{tabular}{|c|c|c|c|c|c|}
\hline \multirow{2}{*}{ Amostra } & \multirow{2}{*}{ Adicionado } & \multicolumn{2}{|c|}{$\begin{array}{c}\text { Rutina (mg mL }{ }^{-1} \text { ) } \\
\text { Biossensores }\end{array}$} & \multicolumn{2}{|c|}{$\begin{array}{c}\text { Recuperação } \\
(\%)\end{array}$} \\
\hline & & (I) & (II) & (I) & (II) \\
\hline \multirow{3}{*}{ A } & 14,41 & $14,02 \pm 0,04$ & $13,72 \pm 0,03$ & 97,3 & 95,2 \\
\hline & 21,62 & $22,16 \pm 0,05$ & $21,20 \pm 0,01$ & 102,5 & 98,1 \\
\hline & 28,82 & $28,64 \pm 0,03$ & $28,90 \pm 0,01$ & 99,4 & 100,3 \\
\hline \multirow{3}{*}{ B } & 14,41 & $13,69 \pm 0,02$ & $14,52 \pm 0,05$ & 95,0 & 100,8 \\
\hline & 21,61 & $21,62 \pm 0,01$ & $21,59 \pm 0,03$ & 100,0 & 99,9 \\
\hline & 28,82 & $29,18 \pm 0,02$ & $28,79 \pm 0,02$ & 101,2 & 99,9 \\
\hline \multirow{3}{*}{$\mathrm{C}$} & 14,41 & $13,26 \pm 0,04$ & $14,22 \pm 0,06$ & 92,0 & 98,7 \\
\hline & 21,61 & $19,62 \pm 0,02$ & $21,90 \pm 0,01$ & 90,8 & 101,3 \\
\hline & 28,82 & $30,27 \pm 0,03$ & $29,80 \pm 0,05$ & 105,0 & 103,4 \\
\hline
\end{tabular}

Tabela 4. Determinação de rutina em produtos farmacêuticos usando os biossensores e o método oficial ${ }^{38}$

\begin{tabular}{lccccc}
\hline \multirow{2}{*}{ Amostra } & \multicolumn{3}{c}{ Rutina $\left(\mathrm{mg} \mathrm{L}^{-1}\right)$} & \multicolumn{2}{c}{ Erro Relativo (\%) } \\
& $\begin{array}{l}\text { Método } \\
\text { oficial* }\end{array}$ & Biossensores & & \\
& (I) & (II) & (I) & (II) \\
\hline A & $19,8 \pm 0,1$ & $21,1 \pm 0,2$ & $20,1 \pm 0,3$ & $+6,6$ & $+1,5$ \\
B & $20,8 \pm 0,2$ & $20,8 \pm 0,2$ & $19,5 \pm 0,1$ & 0,0 & $-6,3$ \\
C & $20,4 \pm 0,1$ & $21,0 \pm 0,3$ & $19,8 \pm 0,1$ & $+2,9$ & $-3,0$ \\
\hline
\end{tabular}

*Método oficial $v s$ biossensores, $\mathrm{n}=6$

nas amostras obtidas empregando-se os biossensores propostos pelo método de adição de padrão e os teores determinados empregando-se o método espectrofotométrico da AOAC. ${ }^{38}$ Os teores de rutina encontrados utilizando o procedimento proposto estão em boa concordância com os teores encontrados empregando-se o método oficial, a um nível de confiança de $95 \%$ e dentro do intervalo de erro aceitável.

\section{CONCLUSÕES}

As micropartículas de quitosana reticuladas com TPP e glioxal apresentaram boa eficiência como suporte na imobilização da lacase. Os biossensores contendo lacase imobilizada em micropartículas obtidas 
por spray drying apresentaram resultados satisfatórios na determinação de rutina em amostras de produtos farmacêuticos, não sofrendo interferência dos excipientes encontrados nas matrizes das amostras. Os sensores desenvolvidos apresentaram linearidade nas mesmas faixas de concentração, entretanto, uma comparação das curvas analíticas, mostra que o biossensor (II) apresentou maior sensibilidade na primeira região linear. Os biossensores propostos apresentaram alta sensibilidade, boa reprodutibilidade, baixo limite de detecção, resposta rápida, excelente estabilidade e simplicidade na construção.

\section{AGRADECIMENTOS}

À Novozymes e ao suporte financeiro do CNPq (Processos $\mathrm{n}^{\circ}$ 472169/2004-1 e 472541/2006-4), pela bolsa de PDJ de I. R. W. Z. de Oliveira e de mestrado de S. C. Fernandes.

\section{REFERÊNCIAS}

1. Ré, M. I.; Drying Technology 1998, 16, 1195.

2. Wendel, S. C.; Çelik, M.; Pharm. Technol. 1997, 21, 124.

3. Sparks, R. E.; Microencapsulation, Wiley \& Sons: New York, 1985, p. $762-763$

4. Pszczola D. E.; Crit. Rev. Food Sci. Nutrition 1993, 33, 501.

5. Davis, S. S.; Illum, L.; Biomaterials 1988, 9, 111.

6. Than, R.; Feldmann, A.; Krebs, B.; Coord. Chem. Rev. 1999, 182, 211.

7. Li, S.; Hu, J.; Liu, B.; Biosystems 2004, 77, 25.

8. Wu, S.; Liu, B.; Li, S.; Int. J. Biol. Macromol. 2005, 37, 263.

9. Mitic, N.; Smith, S. J.; Neves, A.; Guddat, L. W.; Gahan, L. R.; Schenk, G.; Chem. Rev. 2006, 106, 3338.

10. Oliveira, I. R. W. Z.; Fernandes, S. C.; Vieira, I. C.; J. Pharm. Biomed. Anal. 2006, 41, 366

11. Oliveira, I. R. W. Z.; Vieira, I. C.; Enzyme Microb. Technol. 2006, 38, 449.

12. Oliveira, I. R. W. Z.; Vieira, I. C.; Lupetti, K.O.; Fatibello-Filho, O.; Fávere, V. T.; Laranjeira, M. C. M.; Anal. Lett. 2004, 15, 3111.

13. Oliveira, I. R. W. Z.; Vieira, I. C.; Quim. Nova 2006, 29, 932.

14. Fernandes, S. C.; Oliveira, I. R. W. Z.; Vieira, I. C.; Enzyme Microb. Technol. 2007, 40, 661.

15. Rosatto, S. S.; Freire, R. S.; Duran, N.; Kubota, L. T.; Quim. Nova 2001, 24,77 .

16. Leite, O. D.; Lupetti, K. O.; Fatibello-Filho, O.; Vieira, I. C.; Barbosa, A. M.; Talanta 2003, 59, 889.

17. Leite, O. D.; Fatibello-Filho, O.; Barbosa, A. M.; J. Braz. Chem. Soc. 2003, 14, 297.

18. Yaropolov, A. I.; Skorobogatko, O. V.; Vartanov, S. S.; Varfolomeyer, S. D.; Appl. Biochem. Biotechnol. 1994, 49, 257.

19. Sigoillot, C.; Record, E.; Belle, V.; Robert, J. L.; Levasseur, A.; Punt, P. J.; van den Hondel, C. A. M. J. J.; Fournel, A.; Sigoillot, J. C.; Asther, M.; Appl. Microbiol Biotechnol 2004, 64, 346.
20. Liu, Y.; Qu, X.; Guo, H.; Chen, H.; Liu, B.; Dong, S.; Biosens. Bioelectron. 2006, 21, 2195.

21. Vianello, F.; Ragusa, S.; Cambria, M. T.; Rigo, A.; Biosens. Bioelectron. 2006, 21, 2155.

22. Tang, H.; Zhang, W.; Genga, P.; Wang, Q.; Jin, L.; Wu, Z.; Lou, M.; Anal. Chim. Acta 2006, 562, 190.

23. Roy, J. J.; Abraham, T. E.; Abhijithb, K. S.; Kumar, P. V. S.; Thakur, M. S.; Biosens. Bioelectron. 2005, 21, 206.

24. Gomes, S. A. S. S.; Nogueira, J. M. F.; Rebelo, M. J. F.; Biosens. Bioelectron. 2004, 20, 1211.

25. Calabrò, M. L.; Tommasini, S.; Donato, P.; Stancanelli, R.; Raneri, D.; Catania, S.; Costa, C.; Villari, V.; Ficarra, P.; Ficarra, R.; J. Pharm. Biomed. Anal. 2005, 36, 1019.

26. Hasumura, M.; Yasuhara, K.; Tamura, T.; Imai, T.; Mitsumori, K.; Hirose, M.; Food Chem. Toxicol. 2004, 42, 439.

27. Wang, Q.; Ding, F.; Li, H.; He, P.; Fang, Y.; J. Pharm. Biomed. Anal. 2003, 30, 1507.

28. Hassan, H. N. A.; Barsoum, B. N.; Habib, I. H. I.; J. Pharm. Biomed. Anal. 1999, 20, 315.

29. Lavanya, K.; Baggi, T. R.; Microchem. J. 1990, 41, 126.

30. Sakamoto, M.; Takamura, K.; Microchem. J. 1978, 23, 374.

31. Sun, Y.; Guo, T.; Sui, Y.; Li, F. M.; J. Sep. Sci. 2003, 26, 1203.

32. Chen, G.; Zhang, H. W.; Ye, J. N.; Anal. Chim. Acta 2000, 423, 69

33. Kang, J. W.; Lu, X. Q.; Zeng, H. J.; Liu, H. D.; Lu, B. Q.; Anal. Lett. 2002, 35, 677 .

34. Jarosz-Wilkolazka, A.; Ruzgas, T.; Gorton, L.; Enzyme Microb. Technol. 2004, 35, 238 .

35. Blasco, A. J.; González, M. C.; Escarpa, A.; Anal. Chim. Acta 2004, 511, 71.

36. Couto, S. R.; Herrera, J. L.T.; Biotechnol. Adv. 2006, 24, 500.

37. Minussi, R. C.; Pastore, G. M.; Durán, N.; Trends Food Sci. Technol. 2002, 13, 205.

38. Official Methods of Analysis of AOAC International, $16^{\text {th }}$ ed., 1995, p. 29.

39. Shu, X. Z.; Zhu, K. J.; Int. J. Pharm. 2002, 233, 217.

40. Desai, K. G. H.; Park, H. J.; J. Microencapsulation 2005, 22, 377.

41. Corrigan, D. O.; Healy, A. M.; Corrigan, O. I.; Eur. J. Pharm. Biopharm. 2006, 62, 295.

42. Wang, H. S.; Pan, Q. X.; Wang, G. X.; Sensors 2005, 5, 266.

43. Hung, T. C.; Giridhar, R.; Chiou, S. H.; Wu, W. T.; J. Mol. Catal. B: Enzym. 2003, 26, 69 .

44. Betigeri, S. S.; Neau, S. H.; Biomateriais 2002, 23, 3627.

45. Gomes, S. A. S. S.; Rebelo, M. J. F.; Sensors 2003, 3, 166.

46. Abdullah, J.; Ahmad, M.; Heng, L. Y.; Karuppiah, N.; Sidek, H.; Sensors 2007, 7, 2238

47. Freire, R. S.; Duran, N.; Kubota, L. T.; Anal. Chim. Acta 2002, 463, 229.

48. Yaropolov, A. I.; Shleev, S. V.; Morozova, O. V.; Zaitseva, E. A.; MarkoVarga, G.; Emneus, J.; Gorton, L.; J. Anal. Chem. 2005, 6, 553. 